\title{
COMPUTER AIDED DETECTION OF PROSTATE CANCER USING FUSED INFORMATION FROM DYNAMIC CONTRAST ENHANCED AND MORPHOLOGICAL MAGNETIC RESONANCE IMAGES
}

\author{
D. Ampeliotis ${ }^{\dagger}$, A. Antonakoudi ${ }^{\dagger}, K$. Berberidis $^{\dagger}$, E. Z. Psarakis ${ }^{\dagger}$ \\ ${ }^{\dagger}$ Computer Engineering and Informatics Department, University of Patras, 26500, Rio-Patras, Greece \\ ${ }_{\ddagger}^{\ddagger}$ Computing and Information Systems Department, The Philips College, 28008, Nicosia, Cyprus
}

\begin{abstract}
This paper presents a computer-aided diagnosis scheme for the detection of prostate cancer. The pattern recognition scheme proposed, utilizes fused dynamic and morphological features extracted from magnetic resonance images (MRIs). The performance of the proposed scheme has been evaluated through extensive training and testing on several patient cases, where the staging of their condition has been previously evaluated by both ultrasoundguided biopsy and radiological assessment. The classification scheme is based on Probabilistic Neural Networks (PNNs), whose parameters are estimated using the Expectation-Maximization (EM) algorithm during a training phase. Fusion of the image characteristics is performed by properly aligning the respective T1-weighted dynamic and T2weighted morphological images, allowing accurate feature selection from both images. The proposed classification scheme as well as the effect of fusion on the extracted features is tested, with respect to the correct classification rate (CCR) of each case.
\end{abstract}

Index Terms - Pattern recognition, Biomedical magnetic resonance imaging, Neural network applications

\section{INTRODUCTION}

In recent years, prostate cancer has been established as the third cause of cancer-related mortality in developed countries with approximately $10 \%$ of men suffering from the disease [1]. Early detection of prostatic carcinoma is very important since prostate cancer is better treated at early stages of the disease. Tests such as the prostate specific antigen test (PSA), and digital rectal examination (DRE) are at present the best known markers for early diagnosis of prostate cancer. However, none of them offers accurate information about the location and extend of the lesion(s), unless a radical prostatectomy is performed on the patient. Biopsy, a rather painful and invasive examination, performed on regions of interest (ROI) in the prostate gland

This research was supported by the Cyprus Research Promotion Foundation, by research contract PLHRO/0104/03 offers more accurate results through histological analysis as far as the extend and location of the tumor is concerned. However, it has been reported that almost a $10 \%$ of incipient cancers could go undetected due to the inability of the needle to puncture at the precise location of the tumor.

Recently, several non-invasive imaging techniques such as ultrasound (TRUS) and magnetic resonance imaging (MRI) have been used for prostate cancer diagnosis and staging. High resolution Magnetic Resonance imaging has exhibited a well-known ability in representing a more accurate morphology of soft water-based tissues such as the prostate gland than other methods, making it an increasingly required modality for the detection of prostate carcinomas [2].

The aim of this work is to exhibit the ability of the proposed classification scheme to accurately differentiate between malignant and normal prostatic tissue. This could potentially become an invaluable solution to physicians in their attempt to characterize regions of interest within the prostate which are uncertain and biopsy has failed to give a histological profile. Probabilistic Neural Networks [3], trained using the Expectation Maximization [4] algorithm and a significant number of training vectors of fused MRI characteristics (features), are used to create the corresponding clusters for healthy and malignant tissue.

\subsection{Image Acquisition}

The proposed classification system has been trained using 10 patient cases. The recruited patients were subjected to both ultrasound-guided biopsy and MRI examination for a period of one year. The MR images were acquired by a 1.5 Tesla MRI scanner using a body coil. The prostate gland was segmented in eight MR image slices of a $6 \mathrm{~mm}$ thickness and the distance between slices was $1.2 \mathrm{~mm}$. The types of the collected MR images were T2-weighted morphological images and T1-weighted dynamic contrast enhanced (DCE) images. The patients have been injected with a contrast agent prior to the scanning of the DCE examination. The dynamic images consist of a series of thirty images for each slice of the prostate gland, with a temporal resolution of 9 seconds between them, resulting to a total of 240 images. The spatial resolution of the 


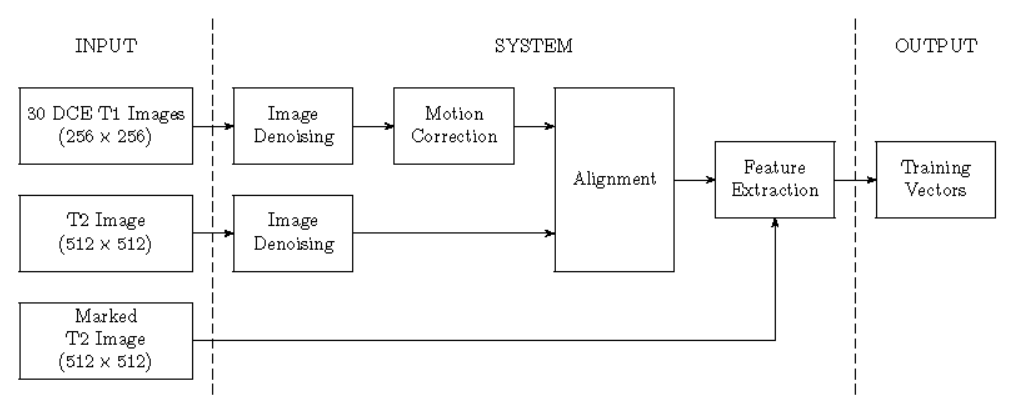

Figure 1. Schematic diagram of the system used for creating the pattern recognition database

DCE images is $1.5 \mathrm{~mm}$ per pixel with $256 \times 256$ pixels per image. The corresponding T2 weighted morphological images were also generated for each of the eight slices, with a spatial resolution of $0.5 \mathrm{~mm}$ per pixel resulting in $512 \times 512$ pixels per image. The lack of equal resolution on morphological and DCE images is due to the fact that the DCE images should provide a sufficient temporal resolution that is traded-off against spatial resolution.

\section{CREATION OF THE DATABASE}

Figure 1 depicts the procedure used for creating the pattern recognition database. The three input components used to create the series of training vectors for a single MR slice of a patient consist of 30 DCE T1-weighted images, the respective T2-weighted image and a marked T2weighted image in which expert radiologists have indicated (marked) ROIs of malignant and/or benign tissue in accordance with the biopsy report. Each of the resulting training vectors corresponds to a single voxel on the MR image within the marked ROI. A wavelet based noise removal algorithm is applied on both the dynamic and morphological images to minimize distorting effects of noise during image acquisition. In the sequel, a motion correction algorithm is applied to the 30 DCE T1-weighted images to reduce the effects of patient/organs motion that may have occurred during image acquisition. The T1weighted and $\mathrm{T} 2$-weighted images are aligned using the method described in Section 2.3. The aligned T1-weighted and $\mathrm{T} 2$-weighted images are finally used to extract features that will be used in the pattern recognition algorithm.

\subsection{Image Denoising}

It is well known that MR images are corrupted by noise mainly due to thermal agitation of electrons or ions of the receiver coil and attached electronics as well as due to the electrolytes in the patient's body [5]. Moreover, due to the signal processing operations that take place in obtaining the magnitude image from its complex valued Fourier transform, it is known that such noise follows the Rice distribution [6]. Thus, Wiener filtering is not the optimal denoising method for MRI. Rather, wavelet domain thresholding techniques are usually employed that offer the increased capability of keeping important image characteristics almost unaltered. However, in such methods, the unknown variance of the noise has to be estimated. In this work, we employed the minimum absolute deviation (MAD) method to tackle this problem. MAD is a standard method that estimates the level of the noise by taking the median of the modulus of the smallest scale wavelet coeffícients [7].

\subsection{Motion Correction}

As already mentioned, the $30 \mathrm{~T} 1$-weighted images are acquired over a time period of 270 seconds. This time interval is large enough so that motion of the patient being examined may have occurred. Furthermore, unintended micro-motion of the patient's internal organs is unavoidable due to organic reasons. Thus, it is expected that the DCE images are not perfectly aligned with each other and that a motion correction algorithm should be applied. However, in this case there are two particularities that should also be taken into account, namely: (a) the motion due to the movement of the patients organs results in non-rigid movement of the images, as different areas of an image suffer different displacement, and (b) as the $30 \mathrm{~T} 1$-weighted images capture the concentration of the contrast agent that varies with time (and area of the image), pixel intensities may change significantly from frame to frame even if they correspond to the same area of the patient's tissue.

Problem (a) is usually addressed by applying a rigid motion estimation algorithm locally, for all possible subimages of given dimensions, and output the estimated transformation to be valid only for the central pixel of each such sub-image. In this work, we adopted the aforementioned methodology in conjunction with a search algorithm for motion estimation. In particular, for each $p \times p$ ( $p$ assumed odd) sub-image, a squared error is computed between all $p \times p$ neighboring (i.e. of relatively small displacement) sub-images in the previous image, and the one for which the error was found to be minimum is selected as the corresponding sub-image that defines the displacement.

Problem (b) however is quite more complicated. In this work, we address this issue by aligning not the original T1-weighted images, but rather, the "normalized spatialgradients" of the T1 images. This is justified based on the assumption that although the intensities of the origi- 
nal images will have time varying characteristics, important features such as edges (which are captured by the spatial-gradient) remain almost constant with time. More specifically, let $\mathbf{I}_{i} i=1,2, \ldots, 30$ denote the 30 DCE images to be aligned with each other and $\mathrm{G}_{i}$ denote the magnitude of the spatial-gradient of $\mathbf{I}_{i}$. Denote also as $G_{i, n, k}$ the $p \times p$ sub-image of $\mathbf{G}_{i}$ whose central pixel has coordinates $[n, k]$. Then, the corresponding normalized image is obtained as

$$
\hat{G}_{i, n, k}[m, q]=\frac{G_{i, n, k}[m, q]-\overline{G_{i, n, k}}}{\left\|G_{i, n, k}-\overline{G_{i, n, k}}\right\|}
$$

where $\overline{G_{i, n, k}}$ is the average of the elements of $G_{i, n, k}$ and || $\cdot \|$ denotes the Euclidean norm. Finally, the "normalized squared error" between two sub-images $G_{i, n, k}$ and $G_{i-1, n^{\prime}, k^{\prime}}$ is defined as

$$
\operatorname{NSE}\left(G_{i, n, k}, G_{i-1, n^{\prime}, k^{\prime}}\right)=\left\|\hat{G}_{i, n, k}-\hat{G}_{i-1, n^{\prime}, k^{\prime}}\right\|^{2}
$$

and the exhaustive search algorithm minimizes this error for $n^{\prime}=n-s, \ldots, n+s$ and $k^{\prime}=k-s, \ldots, k+s$ where the parameter $s$ defines the size of the search area.

\subsection{Alignment of $\mathrm{T} 1$ and $\mathrm{T} 2$ weighted images}

T1 and T2 weighted MRIs use different RF excitation pulses in order to emphasize on different properties of tissues. To obtain T1-weighted contrast, differences in the longitudinal component of magnetization must be emphasized. On the other hand, T2 contrast is revealed if differences in the transverse relaxation times of different tissues become apparent [5]. As a result, T1 and T2 images cannot be aligned using their intensity values, in general.

Thus, to resolve the problem of alignment one must seek for image features that are independent of the particular contrast weighting. In particular, we need to estimate the rotation angle $\theta$, the scaling factor $\alpha$, and the translations $t_{x}$ and $t_{y}$ along each axis, that must be applied to the T1 image so that it becomes aligned to the T2 image.

For the scaling factor $\alpha$, information produced by the MRI scanner was used. More specifically, by dividing the values of the field named "spatial resolution" for the two imaging methods, the value of $\alpha=3$ was found.

For the estimation of the rotation angle $\theta$ and the translation $t_{x}$, we used the fact that MRI images of the prostate are symmetric with respect to an almost vertical axis. In particular, let I denote the image for which we want to estimate its symmetry axis. Let also $\mathbf{I}_{\theta_{0}}$ denote image I after rotation by an angle $\theta_{0}$. Then, the axis defined by the parameters $\left(\theta_{0}, t_{x_{0}}\right)$ may be associated to the cost

$$
S\left(\mathbf{I}, \theta_{0}, t_{x_{0}}\right)=\left\|\mathbf{I}_{\left(\theta_{0}, t_{x_{0}}-K\right)}^{\prime}-\mathbf{I}_{\left(\theta_{0}, t_{x_{0}}+K\right)}\right\|^{2}
$$

where $\mathbf{I}_{\left(\theta_{0}, t_{x_{0}}+K\right)}$ denotes a sub-image of $\mathbf{I}_{\theta_{0}}$ consisting of the columns $t_{x_{0}}+1, \ldots, t_{x_{0}}+K$ (right sub-image) and $\mathbf{I}_{\left(\theta_{0}, t_{x_{0}}-K\right)}^{\prime}$ denotes the sub-image of $\mathbf{I}_{\theta_{0}}$ consisting of columns $t_{x_{0}}-1, \ldots, t_{x_{0}}-K$, i.e. the columns of $\mathbf{I}_{\theta_{0}}$ appear in reverse order. Minimization of (3) with respect to $\theta_{0}, t_{x_{0}}$ can be carried out by direct examination of various angles in the interval $[-\Theta,+\Theta]$ and various integer translations in the interval $[C-L, C+L]$ where $C$ denotes the central column of $\mathbf{I}$. Thus, by estimating the symmetry axes $\left(\theta_{1}, t_{x_{1}}\right)$ and $\left(\theta_{2}, t_{x_{2}}\right)$ of the T1 and T2 images independently, we have that $\theta=\theta_{2}-\theta_{1}$ and $t_{x}=t_{x_{2}}-t_{x_{1}}$.

Finally, for the translation parameter $t_{y}$, we use the scaled by $\alpha$ and symmetric T1-weighted image and the symmetric T2-weighted image. The parameter $t_{y}$ is estimated as the translation that minimizes the squared error between the aforementioned images.

\subsection{Feature Extraction}

Using the aligned $\mathrm{T} 1$ and $\mathrm{T} 2$ weighted images, vectors of features are extracted using the benign and/or malignant areas from the marked T2 image. In particular, for each pixel of the marked $\mathrm{T} 2$ image that belongs to a benign (malignant) area, the respective coordinates of the T1 image are computed. Each vector of features consists of the 30 intensity values from the $30 \mathrm{~T} 1 \mathrm{DCE}$ images at the computed coordinates, the intensity value of the T2-weighted image as well as two spatial moments computed on a $3 \times 3$ area around the current pixel. These two moments are the variance and kurtosis of this $3 \times 3$ area, and they are used to capture possible inhomogeneity of the area. Thus, a total of 33 features are used in each vector of features.

Concerning the diagnostic value of the aforementioned features, the 30 values extracted from the T1 image constitute a dynamic curve, indicating malignancy when the curve exhibits rapid increase (high slope) and then settles to a constant value [8]. The value of the pixel on the T2weighted image indicates cancer when lower (dark-grey) intensities appear as opposed to healthy tissue which returns higher (white) intensities [9]. The variance and kurtosis offer further morphological information in terms of the homogeneity of the suspected area.

\section{PATTERN RECOGNITION}

Probabilistic Neural Networks try to categorize a feature vector $\mathrm{x}$ into two categories $H_{a}$ or $H_{b}$ by implementing the general Bayesian rule

$$
d(\mathbf{x})=\left\{\begin{array}{lll}
H_{a} & \text { if } & p_{a} l_{b} f_{a}(\mathbf{x})>p_{b} l_{a} f_{b}(\mathbf{x}) \\
H_{b} & \text { if } & p_{a} l_{b} f_{a}(\mathbf{x})<p_{b} l_{a} f_{b}(\mathbf{x})
\end{array}\right.
$$

where $p_{a}$ and $p_{b}$ denote the a-priori probabilities of categories $H_{a}$ and $H_{b}$, respectively, and $l_{a}\left(l_{b}\right)$ denotes the cost associated with the decision in favor for $H_{a}\left(H_{b}\right)$ while the actual decision should be in favor of $H_{b}\left(H_{a}\right)$. Also, $f_{a}(\mathrm{x})$ and $f_{b}(\mathbf{x})$ denote the probability density functions of the respective categories.

The parameters $p_{a}, p_{b}, l_{a}, l_{b}$ are usually set by experts about the detection problem at hand. Moreover, the unknown probability density functions of the categories should be estimated using training data. In [10], it is proposed to 


\begin{tabular}{|c|c|c|c|c|c|c|c|c|c|c|c|c|c|c|c|}
\hline & \multicolumn{3}{|c|}{$P_{6}$} & \multicolumn{3}{|c|}{$P_{7}$} & \multicolumn{3}{|c|}{$P_{8}$} & \multicolumn{3}{|c|}{$P_{9}$} & \multicolumn{3}{|c|}{$P_{10}$} \\
\hline & $\overline{\mathrm{T} 1}$ & $\overline{\mathrm{T} 2}$ & $\overline{\bar{F}}$ & $\overline{\text { T1 }}$ & $\overline{\mathrm{T} 2}$ & $\overline{\bar{F}}$ & $\overline{\mathrm{T} 1}$ & $\overline{\mathrm{T} 2}$ & $\overline{\bar{F}}$ & $\overline{\mathrm{T} \text { T1 }}$ & $\overline{\mathrm{T} 2}$ & $\overline{\bar{F}}$ & $\overline{\mathrm{T} \text { T1 }}$ & $\bar{~} \overline{\mathrm{T} 2}$ & $\overline{\bar{F}}$ \\
\hline$P_{1}$ & 73.2 & 82.1 & 81.2 & 63.3 & 79.8 & 85.0 & 89.0 & 84.6 & 89.1 & 84.9 & 88.4 & 90.8 & 56.0 & 84.5 & 82.3 \\
\hline$P_{2}$ & 83.3 & 83.0 & 86.6 & 43.4 & 80.4 & 85.2 & 99.9 & 81.7 & 100.0 & 91.4 & 96.6 & 99.2 & 59.6 & 94.6 & 87.1 \\
\hline$P_{3}$ & 56.8 & 80.3 & 84.4 & 82.6 & 82.3 & 88.0 & 63.3 & 80.6 & 87.6 & 64.5 & 83.1 & 88.3 & 58.8 & 81.5 & 83.3 \\
\hline$P_{4}$ & 57.7 & 45.0 & 72.1 & 56.6 & 61.5 & 82.6 & 74.5 & 45.7 & 73.7 & 72.7 & 54.3 & 78.0 & 55.2 & 47.1 & 76.5 \\
\hline$P_{5}$ & 79.6 & 68.2 & 78.6 & 56.9 & 75.4 & 89.1 & 90.4 & 68.6 & 66.2 & 85.8 & 73.3 & 80.9 & 64.3 & 69.6 & 75.0 \\
\hline
\end{tabular}

Table 1. CCR of the EM-trained PNNs for various training sets. Average CCRs are $70.5 \%, 74.8 \%$ and $83.6 \%$

estimate a general pdf as the average of Gaussian densities. Thus, we may approximate the density $f_{a}(\mathbf{x})$ as

$$
f_{a}(\mathbf{x})=\frac{1}{M_{a}(2 \pi)^{\frac{N}{2}}|\Sigma|} \sum_{i=1}^{M_{\alpha}} e^{-\frac{1}{2}\left(\mathbf{x}-\mathbf{x}_{\alpha_{i}}\right)^{T} \Sigma^{-1}\left(\mathbf{x}-\mathbf{x}_{\alpha_{i}}\right)}
$$

where $\mathbf{x}_{a_{i}}$ denote the mean vectors of each Gaussian (centroids), $\Sigma$ is the $N \times N$ covariance matrix of each Gaussian kernel and $N$ is the length of the input vectors. $M_{a}$ is the number of kernels used, which constitutes also a parameter to be estimated. In this work, the centroids $\mathrm{x}_{a_{i}}, \mathrm{x}_{b_{i}}$ of the categories $H_{a}$ and $H_{b}$ respectively as well as the covariance matrix $\Sigma$ were estimated according to [11].

\section{EXPERIMENTAL RESULTS}

The performance of the scheme implemented was assessed in terms of the Correct Classification Rate and the results obtained are summarized in Table 1. In particular, Table 1 presents the CCR obtained by the classification system for 25 cases. Patients $P_{1}-P_{5}$ have normal tissue while patients $P_{6}-P_{10}$ have malignant areas. Thus, in each case, a pair of patients is excluded from training (one with normal tissue and one having malignant areas) and their data is used for testing. The parameters used for the motion correction algorithm were $p=25$ and $s=2$. Also, the search space for the T1 - T2 alignment algorithm was $\Theta \in\{-2,-0.95,-0.90, \ldots, 2\}$ and $L=20$. For the pattern recognition algorithm, the a-priori probabilities were assumed equal to $1 / 2$ and the respective costs were set equal to unity, i.e. no bias was induced to the classification system. Finally, the numbers $M_{a}$ and $M_{b}$ of the kernels per category used, were selected as $M_{a}=M_{b}=3$.

From Table 1 we observe that, in most cases, the classification system employing fused features obtains better correct classification rates. Furthermore, in the rest cases, the CCR obtained is very close to the best CCR between the two other systems that employ features only from one imaging modality. Also, the average CCR for the three systems shows that classification using fused features outperforms the other approaches by about $10 \%$.

\section{CONCLUSIONS}

In this work, a database of malignant and benign prostate tissue was created. The database involved inform ation both from DCE T1-weighted MRI and from morphological T2weighted MRI. This fusion of information was achieved by properly aligning the images of the two modalities. Probabilistic neural networks, trained using the EM algorithm, were employed for the detection of malignant or benign tissue of the prostate. Experimental results verified that the aforementioned fusion of characteristics increases the CCR of the proposed automatic detection scheme.

\section{REFERENCES}

[1] J. E. S Husband, R. H. Reznek, Imaging in Oncology, Oxford Press, 1998.

[2] Villeirs et.al, "Magnetic resonance imaging anatomy of the prostate and periprostatic area: a guide for radiotherapists", J. of the Eur. Soc. for Therapeutic Radiology, vol. 76, , pp. 99-106, 2005.

[3] Specht D. F., Probabilistic Neural Networks, Neural Networks, vol. 3, 1990, pp. 109-118.

[4] Dempster A. P., Laird N. M. and Rubin D. B. Maximum Likelihood from incomplete data via the EM algorithm, Journal of the Royal Statistical Society (B), $39,1-38,1977$

[5] J. L. Prince and J. M. Links, Medical Imaging Signals and Systems, Pearson Prentice Hall Bioengineering, 2006

[6] R. D. Nowak, "Wavelet Based Rician Noise Removal for Magnetic Resonance Imaging", IEEE Trans. on Image Processing, vol. 8, No. 8, pp. 1408-1419, October 1999.

[7] S. Mallat, A Wavelet Tour of Signal Processing, Academic Press, 1998.

[8] M.R. Engelbrecht et.al, "Discrimination of Prostate Cancer from normal peripheral zone and central gland tissue by using dynamic constrast enhanced MR imaging", J. of Radiology, vol. 229, , pp. 248-254, 2003.

[9] Cruz et.al, "Characterization of low-intensity lesions in the peripheral zone of prostate on pre-biopsy endorectal coil on MR imaging", European Radiology, vol. 12, , pp. 357-365, 2003.

[10] Parzen E., On estimation of probability density function and mode, Annals of Mathematics, 33, pp. 10651076,1962

[11] Streit R. L., and Luginbuhl T. E. Maximum Likelihood Training of Probabilistic Neural Networks, Neural Networks, 3, pp 109-118, 1994 